\title{
Control Strategy of Generators' Dynamic Reactive Power Based on Local Voltage Signal
}

\author{
Qian WANG ${ }^{1,}$ a, Qian $\mathrm{CHENG}^{2}$, Xiaoming $\mathrm{LI}^{2}$, Junhong GUO ${ }^{3}$ \\ ${ }^{1}$ Electric Power Research Institute of Guangdong Power Grid Corporation, Guangzhou 510080, \\ China \\ ${ }^{2}$ School of Electrical Engineering, Northeast Dianli University, Jilin 132012, China \\ ${ }^{3}$ School of Electric Power, South China University of Technology, Guangzhou, 510640, China \\ aemail:wqandwl@gddky.csg.cn
}

Keywords: Dynamic voltage stability; Dynamic reactive power; High side voltage; Excitation control

\begin{abstract}
The main factors of dynamic reactive power output are analyzed theoretically in this paper. A dynamic reactive power control strategy based on local voltage signal is presented. Monitoring the high side voltage online, the power unit which high side voltage is conformed to the preset criterion, is selected to be involved in the excitation control. Thus, the dynamic reactive power control by generator is realized. Combined with the voltage recovery existed in Guangdong power grid in 2014, the control strategy presented in the paper is verified properly and effectively, it is of great value to further improve the dynamic voltage stability of power system.
\end{abstract}

\section{Introduction}

In recent years, due to the impact of the electrical factors, such as rapid growth of load, dense placement of large-capacity of HVDC converter station, cascading failure of large area interconnected power systems and mass flow transferring after lockup fault condition of DC system on the modern power grid, dynamic voltage stability after fault has become increasingly prominent, which is also an important constraint to the safe and stable operation of interconnected power grid [1].

The characteristics of voltage recovery and stability after fault are mainly determined by dynamic reactive power support, it produces extraordinary significance technically on enhancing the capability of resisting failure and ensuring the stable operation of power grid by providing adequate dynamic reactive power support [2]. Generator, as a vital source of dynamic reactive power, is characterized with rapid reactive adjustment and non-additional investment [3]. The main influence on the system dynamic voltage stability brought by STATCOM is analyzed by [4]. The significant implication of the load compensation coefficient optimization in generator excitation control on improving the dynamic voltage stability is verified through simulation in [5]. In [6], excitation system optimization model is established to analyze the impact of different coefficients on the voltage levels respectively, and it is verified that this optimization method can improve the voltage levels obviously.

Control strategy of power system dynamic voltage stability based on local voltage signal is proposed in this paper, through monitoring the generator high side bus voltage online, central controller sends control signals to excitation controller, and then the generator can output more dynamic reactive power in order to improve the power system voltage stability when the high side voltage is conformed to the preset criterion.

\section{Impact factors of generator dynamic reactive power output}

To ensure the operating voltage with normal levels, the power system must have sufficient reactive power to meet the demand that the load and power grid need. After fault, generator can give full play to its ability of reactive power output based on the changes of terminal voltage. Setting the terminal voltage as the reference phase, the complex power of generator is written as (1). 
$\bar{S}=P+j Q=\dot{E}_{q} \hat{I}=\frac{E_{q} U}{X_{d}} \sin \delta+j\left[\frac{E_{q}^{2}-E_{q} V_{g} \cos \delta}{X_{d}}\right]$

The reactive power is as (2):

$Q=\frac{E_{q}^{2}-E_{q} V_{g} \cos \delta}{X_{d}}$

where $E_{q}$ is excitation voltage, namely generator unloaded voltage, which is proportional to excitation current; $V_{g}$ is terminal voltage; $X_{d}$ is synchronous reactance of implicit pole synchronous motor; $\delta$ is power angle. Equation (2) shows that the terminal voltage drop after fault, it would be maintained at a given level by increasing the unloaded voltage $E_{q}$ to improve the generator reactive power output [7].

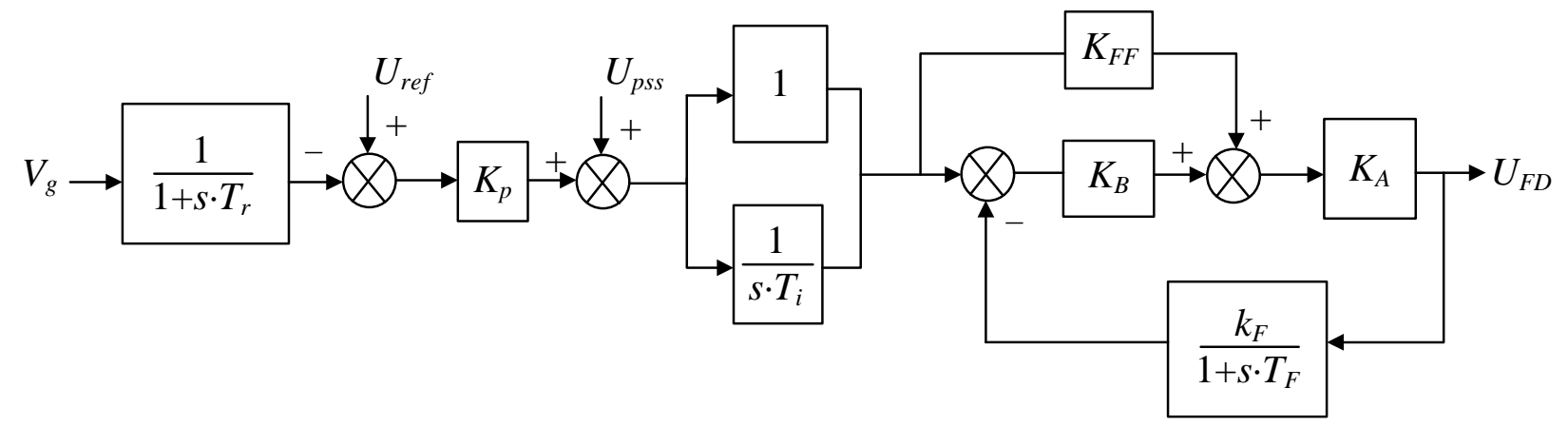

Fig 1. Generator excitation system model

The reactive power can be regulated by synchronous generator through adjusting the excitation current. In Fig. 1, when the excitation reference voltage $U_{\text {ref }}$ increases, excitation voltage $E_{f d}$, namely $E_{q}$ will also increases, thus leading to the increase of excitation current, further increasing the reactive power output. Therefore, when the excitation current increases, terminal voltage vectors are shown as Fig. 2.

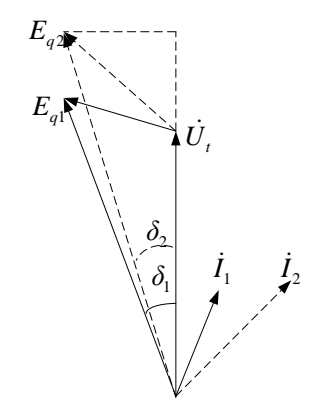

Fig 2 Terminal voltage vectors when the excitation current increases

Generator unloaded voltage increases while the excitation current is increasing. In Fig. 2 shows the variation of vectors $E_{q}$, excitation current $I$ and $\delta$. For the solid vectors are the state 1 and the dash vectors are the state2. If the terminal voltage $U$ is constant, as long as the unloaded voltage $E_{q}$ increases or power angle $\delta$ decreases, the output of generator reactive power will be increased. In connection with Fig. 1, it is clear that the change of excitation reference voltage $U_{\text {ref }}$ would exert an impact on the output of generator reactive power.

\section{Generator excitation control strategy based on high side bus voltage}

\section{The impact of high side bus voltage on the power system voltage stability}

From the relationship between reactive power and voltage, the transmission of reactive power is mainly determined by the voltage amplitude. To maintain a favorable voltage distribution and a high voltage level at the same time, it is necessary to take all measures about reactive power compensation to improve the system voltage level. 


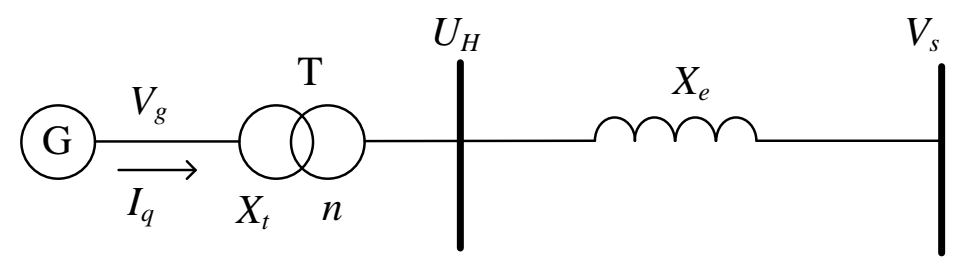

Fig 3 Single-machine infinite model

where in the Fig. 3 is a single-machine infinite model, the related parameters or presets are all under the ideal condition. $V_{g}$ refers to the terminal voltage; $\underline{I}_{q}$ refers to reactive current; $X_{t}$ refers to reactance of step-up transformer; $V_{H}$ refers to the high side voltage of step-up transformer; $X_{e}$ refers to the reactance of transmission line; $V_{s}$ refers to the infinite system voltage.

Considering that generator will output reactive power with the normal operation of power system, that is, $Q>0$, according to Fig. 3:

$V_{H}=\frac{V_{g}^{2}-X_{t} Q}{V_{g} \cos \delta}$

where $Q$ represents the generator's reactive power. Since the value of $X_{t}$ is to some extend small, and it considers that when $\delta$ is approximately equal to 0 , (3) can be transferred into (4):

$V_{H}=V_{g}-\frac{X_{t} Q}{V_{g}}$

The generator terminal voltage will drop after fault, and then it is deviated from the system voltage, in (4), the high side voltage of the generator step-up transformer will also drop at this time. Therefore, the power system voltage can be maintained with a high level by controlling the generator high side voltage, thus contributes to improve the system dynamic voltage stability.

The emergency control method of generator reactive power based on high side voltage

Considering the system voltage recovery problems, an emergency control method of generator reactive power output based on local voltage signal is proposed in this paper, thus, to provide sufficient dynamic reactive power support for voltage dropping and improve the system dynamic voltage stability.

After fault, the control strategy of generators' dynamic reactive power is conducted to tackle the problem of voltage sag. Firstly, the power plant included in excitation control can be chosen effectively and quickly by monitoring the high side bus voltage online. When the voltage of the power plant meet the preset criterion (which is formulated in the following paragraphs), control signals are sent by central controller to the excitation controller, so that it modifies the generator reactive power output to improve the bus voltage recovery.

The generator reactive power output is increased by implementing excitation reference voltage step control, while in actual operation, the limits of generator reactive power output needs to be taken into consideration, provided that the maximum reactive power is $Q_{i \max }$, which is shown as (5):

$$
Q_{\mathrm{i}} \leq Q_{\text {imax }}
$$

Simultaneously, the limits of terminal voltage also needs to be considered, as (6) shows: $V_{\mathrm{i}} \leq V_{i \max }$

Power plants involved in excitation control are chosen determined by the degree of the high side voltage drop. In this paper, the voltage falling percentage $T$ and voltage falling rate $K_{D}$ are defined to indicate the voltage falling degree, expressed as follow equations:

$$
\begin{aligned}
& T=\frac{V_{0}-V_{D \min }}{V_{0}} \times 100 \% \\
& K_{D}=\frac{\Delta V}{\Delta \mathrm{t}}=\frac{V_{0}-V_{D \min }}{t_{D}-t_{0}}
\end{aligned}
$$

where $V_{0}$ refers to the initial value of high side voltage; $V_{D \text { min }}$ refers to the minimum value of high side voltage falling after fault; $t_{0}$ refers to the fault time; $t_{D}$ refers to the time that the voltage falls to 
the minimum value $V_{D \text { min }}$. As is written in (7) and (8), the greater the $T$ is, the shorter that the electrical distance between power plant and fault is; and the greater $K_{D}$ is, the faster that the high side voltage drops. Setting $T_{0}$ and $K_{0}$ as the standard value, after fault, when the voltage falling percentage $T$ and voltage falling rate $K_{D}$ of some plants' high side voltage are respectively greater than $T_{0}$ and $K_{0}$, and then this power plant would prefer to be applied in implementing excitation control.

Notice that the capacity of units involved in excitation control also needs to be taken into account, here parameter $C$ is defined to represent the capacity of the power unit. The outlet of reactive power form the generator would increase following the power unit capacity $C$. Taking $C_{S}$ as the units' standard capacity involved in excitation control, that unit which capacity is smaller than $C_{S}$ cannot be chosen to implement excitation control.

From what has been illustrated above, the flow chart of emergency control strategy of dynamic reactive power based on high side bus voltage of this paper is shown as Fig. 4.

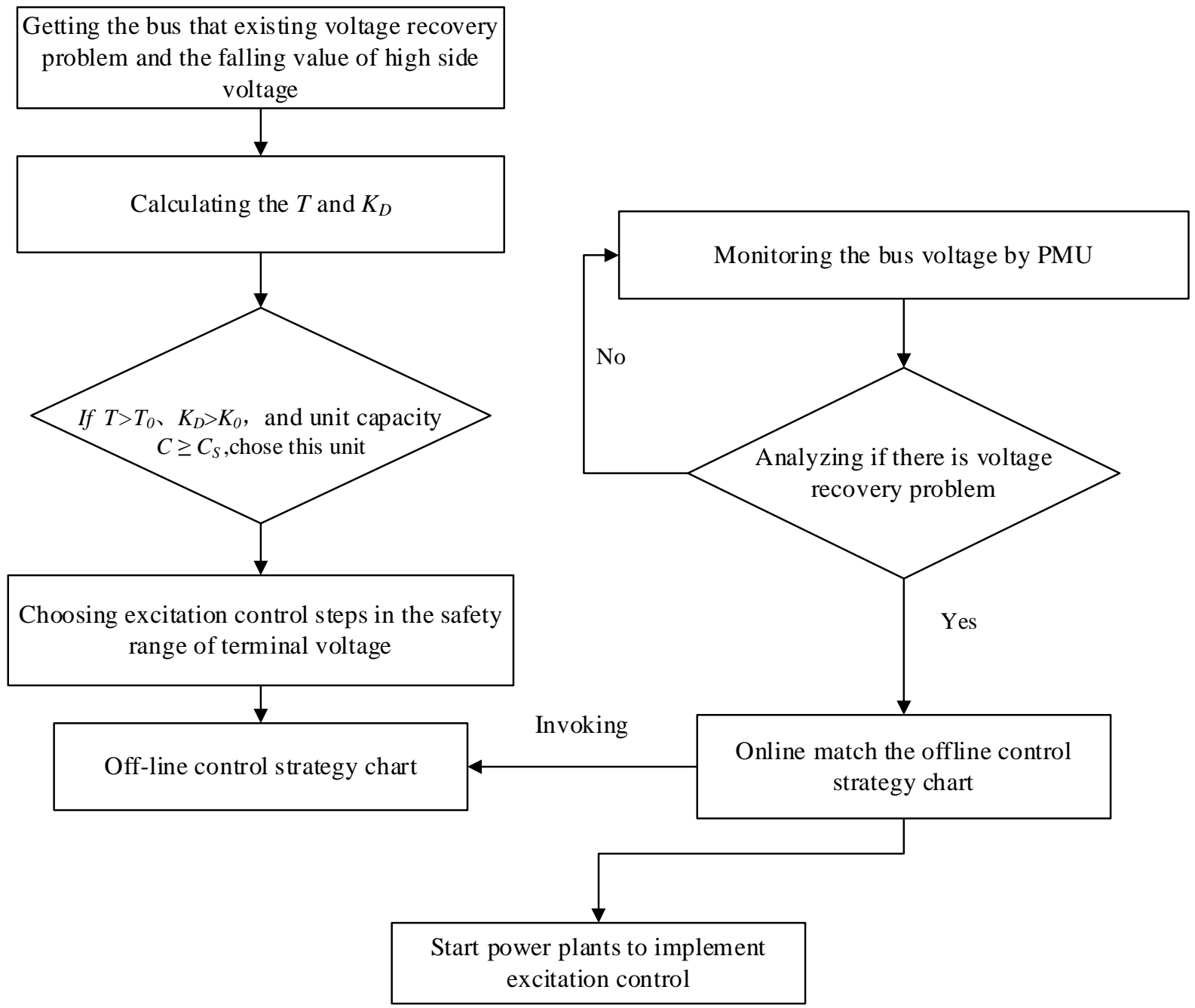

Fig 4 Flow chart of dynamic reactive power control strategy

\section{Case simulation}

In this section, it presents scenario that the Guangdong power grid in 2014 simulation results show voltage delayed problems under a three-phase short circuit fault. Near Shuixiang substation, there existed the problem of voltage delayed recovery in $220 \mathrm{kV}$ Banqiao substation, Chenwu substation and Jinpu substation. And the simulation voltage profiles are shown in Fig. 5. 


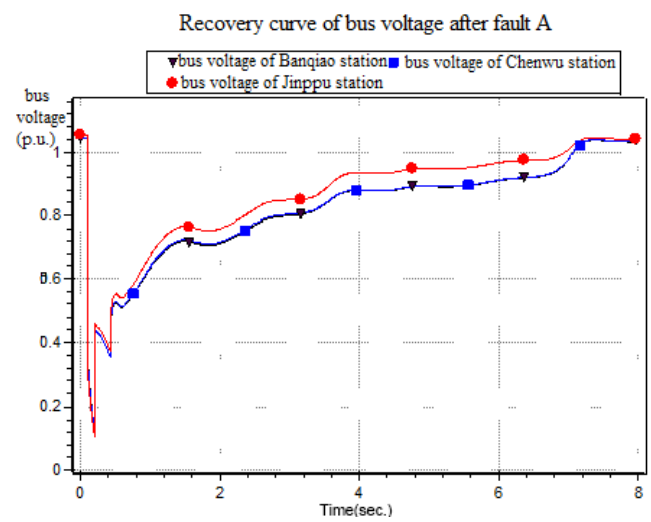

Fig 5.Voltage profiles of 220kV Banqiao, Chenwu and Jinpu station after fault A

As shown in Fig. 5, Banqiao substation kept low voltage for a long time, which was down below to 0.75 p.u. in duration of up to 120 cycles, and was down below to 0.95 p.u. in duration of 340 cycles.

Through analyzing a lot of simulation results, " $T>25 \%$ " and " $K_{D}>3.5$ " are selected as the criteria for determining which units are involved in excitation control. Taking the units' capacity into consideration, $300 \mathrm{MW}$ is set as the standard capacity in this case. Voltage falling percentage $T$ and voltage falling rate $K_{D}$ of all units in Guangdong province are calculated through simulation calculation. The units that satisfy the criteria are listed in Table 1

Table 1 Units that meet the preset criterion

\begin{tabular}{|c|c|c|c|}
\hline Units & $C[\mathrm{MW}]$ & $T$ & $K_{D}$ \\
\hline Guangxu & 600 & $72.55 \%$ & 7.691 \\
\hline Shajiao B & 700 & $72.11 \%$ & 7.647 \\
\hline Shajiao A & 420 & $71.87 \%$ & 7.638 \\
\hline Zhongxin & 600 & $64.18 \%$ & 6.864 \\
\hline Hengyun D & 600 & $62.29 \%$ & 6.661 \\
\hline Huixu & 600 & $42.31 \%$ & 4.456 \\
\hline Pingshi B & 300 & $35.91 \%$ & 3.822 \\
\hline
\end{tabular}

In principle, the greater that the steps of excitation reference voltage is, and the more reactive power that the generator units will export. In addition, the steps should be chosen based on the safety range of terminal voltage. As depicted in Fig. 6, when the steps is set as $3 \%$ or $4 \%$, terminal voltage of Shajiao B Plant \# 2 has exceeded 1.05 p.u., while $2 \%$ steps is more appropriate, and therefore it is selected to be applied in this case.

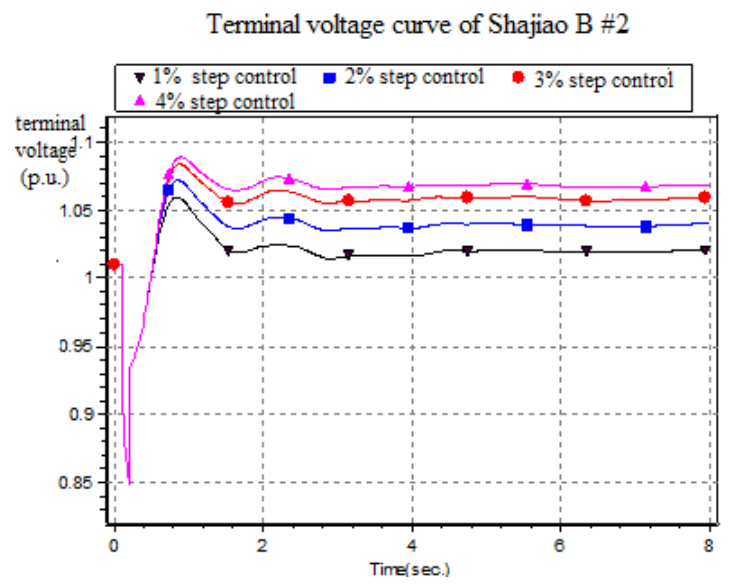

Fig 6. Terminal voltage of Shajiao B \#2 under different steps

According to Table 1, all generator units implement the $2 \%$ excitation step control. To compare the simulation results that voltage curve within or without excitation control, both voltage profile curves are shown as Fig. 7. 


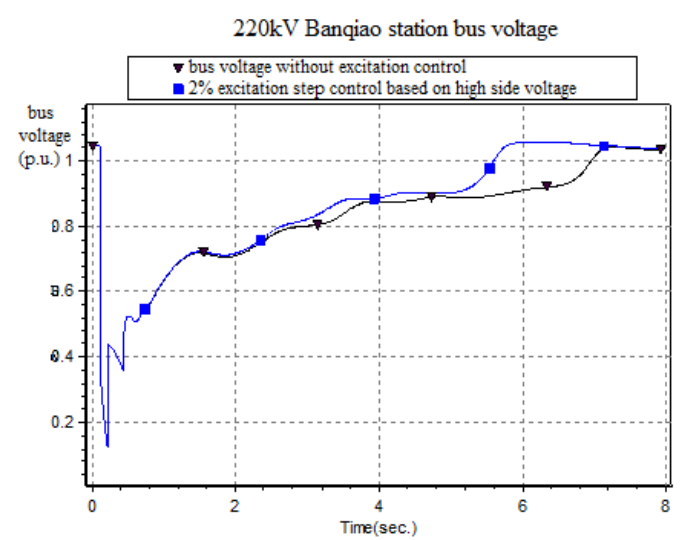

Fig 7.220kV Banqiao station bus voltage before and after excitation control after fault A

According to the simulation results, the time that Banqiao station costs to recover to 0.95p.u. is shortened to $5.48 \mathrm{~s}$, which is $1.24 \mathrm{~s}$ shorter than that without excitation control, and improves the bus voltage recovery time. The above simulation results show that the control strategy proposed in this paper performs well in accurately selecting the units involved in excitation control.

\section{Conclusion}

In this paper, a dynamic voltage stability control strategy based on high side voltage is proposed. When the voltage meets the preset criteria, and then control signals are sent to the excitation controller, thus to realize the generator dynamic reactive power control. In 2014-Guangdong power grid limit operation model, a three-phase short circuit happened in Shuixiang-Guancheng line is analyzed simultaneously, the simulation results show that the control strategy can select the units involved in excitation control accurately, and it creates significance to improve the system dynamic voltage stability.

\section{References}

[1] Guoliang Zhao, Yuanzhang Sun, Lin Cheng, etc. Influences of dynamic voltage stability on power transmission capability of south china power grid from west China to east China [J].Power System Technology, 2004, 28(14): 1-4.

[2] Mingbo Liu, Jinghui Cheng, Ying Cheng. Dynamic voltage stability analysis of parallel AC/DC power systems [J]. Automation of Electric Power System, 1999, 23(16): 27-31.

[3] Yong Tang, Wuzhi Zhong, Huadong Sun, etc. Study on mechanism of power system voltage stability [J]. Power System Technology, 2010, 34(4): 24-29.

[4] Yongchun Su, Shijie Cheng, Jinyu Wen. Reactive power generation management for improvement of voltage stability margine [J]. Electric Power Automation Equipment, 2007, 6(27): 39-42.

[5] C. M. AFFONSO, L. C. SILVA, F. G. LIMA, et al. MW and Mvar management on supply and demand side for meeting voltage stability margin criteria [J]. IEEE Trans on Power System, 2004, 19(3): 1538-1545.

[6] Ming Ye, Shaojie Qu. A survey on reactive power planning research considering voltage stability constraints [J]. Guangdong Electric Power, 2008, 21(9): 6-10.

[7] P. Kundur, J. Paserba, V. Ajjarapu, et al. IEEE/CIGRE joint task force on stability terms and definitions. Definition and Classification of Power System Stability [J]. IEEE Trans on Power System, 2004, 19(2): 1387-1401. 